\title{
Energy Current Rectification and Mobility Edges
}

\author{
Vinitha Balachandran, ${ }^{1}$ Stephen R. Clark, ${ }^{2,3,4}$ John Goold, ${ }^{5}$ and Dario Poletti ${ }^{1}$ \\ ${ }^{1}$ Science and Math Cluster and EPD Pillar, Singapore University of Technology and Design, 8 Somapah Road, 487372 Singapore \\ ${ }^{2}$ H.H. Wills Physics Laboratory, University of Bristol, Bristol BS8 1TL, United Kingdom \\ ${ }^{3}$ Department of Physics, University of Bath, Claverton Down, Bath BA2 7AY, United Kingdom \\ ${ }^{4}$ Max Planck Institute for the Structure and Dynamics of Matter, University of Hamburg CFEL, Hamburg 22761, Germany \\ ${ }^{5}$ School of Physics, Trinity College Dublin, Dublin 2, Ireland
}

(Received 3 October 2018; revised manuscript received 4 March 2019; published 10 July 2019; corrected 19 July 2019)

\begin{abstract}
We investigate how the presence of a single-particle mobility edge in a system can generate strong energy current rectification. Specifically, we study a quadratic bosonic chain subject to a quasiperiodic potential and coupled at its boundaries to spin baths of differing temperature. We find that rectification increases by orders of magnitude depending on the spatial position in the chain of localized eigenstates above the mobility edge. The largest enhancements occur when the coupling of one bath to the system is dominated by a localized eigenstate, while the other bath couples to numerous delocalized eigenstates. By tuning the parameters of the quasiperiodic potential it is thus possible to vary the amplitude, and even invert the direction, of the rectification.
\end{abstract}

DOI: 10.1103/PhysRevLett.123.020603

Introduction.-The quest for sustainable energy has been boosted by the field of quantum thermodynamics [1,2]. Controlling energy transport and conversion at nanoscale is key to the design of future chips, thermoelectric devices, and also quantum computers. One particular class of systems which has attracted considerable attention are current rectifiers, i.e., systems for which the current flows preferentially in one direction [3-14].

Quantum effects can play an important role in the transport properties of a system. For example, noninteracting systems can be current rectifiers when the baths have particles or excitations with different quantum statistics from that of the system which connects them [13]. Interference also plays an important role. For example, Anderson showed that because of interference, a onedimensional disordered material can be an insulator, independent of the magnitude of disorder [15]. Disorder can be mimicked by quasiperiodic potentials like the Aubry-André-Harper (AAH) model, in which the period of the potential is incommensurate with the lattice confining the particles, however with an important difference: the transition between the metallic phase (in which all the eigenstates are delocalized) to the insulating phase (in which all the eigenstates are exponentially localized) occurs at a finite magnitude of the quasiperiodic potential $[16,17]$. Moreover, modifying the potential in the standard AAH model, one can turn the system to be an energy filter because of the presence of mobility edges, i.e., threshold energy values that separate localized from delocalized eigenstates $[18,19]$. Although the AAH model is currently not realistic for a solid-state system, it has been realized with ultracold atoms both for noninteracting and interacting systems [20-25], also in a modified version with mobility edges [26-28].

Modified AAH models with a mobility edge have been shown to display a transition between ballistic transport to insulating behavior separated by a critical line with subdiffusive transport [29]. Since the mobility edge acts as an energy filter, a characteristic that improves the performance of thermoelectric systems, it is important to study whether the mobility edge can strongly affect the rectifying properties of the system [30]. This is what we do in this work. We consider a quadratic bosonic chain with a generalized AAH potential possessing a mobility edge. Since the quasi-periodic potential breaks the spatial reflection symmetry, we only need to use baths with different statistics $[13,14]$ in order to have rectification. Here we consider a bosonic system coupled to spin baths. A proof of principle experimental implementation could be a chain of evanescently coupled cavities with the boundary cavities containing atomic ensembles mimicking the spin baths [31,32].

We will show in the following that the presence of a bulk mobility edge can result in strong rectification when localized eigenstates cluster at one edge of the system connected to one bath. Consequently, drastically different nonequilibrium steady states (NESS) are generated in forward or reverse bias of the applied bath temperatures. The direction of the rectification can be controlled by tuning the quasiperiodic potential parameters that shift the spatial position of the extensive large number of localized modes. While the rectification is strongest at large temperature differences, we show the robustness of the effect by considering different temperatures. 
Model.-We study a one-dimensional generalized AAH model of $L$ noninteracting bosonic modes with on-site modulation $V_{l}$ described by the Hamiltonian

$$
H=-t \sum_{l=1}^{L-1}\left(a_{l}^{\dagger} a_{l+1}+a_{l+1}^{\dagger} a_{l}\right)+\sum_{l=1}^{L} V_{l} a_{l}^{\dagger} a_{l},
$$

where the operators $a_{l}\left(a_{l}^{\dagger}\right)$ annihilates (creates) a boson at site $l$. In the following we consider a large system with $L=1000$ sites; however since the system is ballistic the system size does not play a crucial role. In Eq. (1), $t$ is the hopping parameter, $V_{l}=\mu+2 \lambda\{[1-\cos (2 \pi l b+\phi)] /$ $[1+\alpha \cos (2 \pi l b+\phi)]\}$ [33] is the potential and it is characterized by the deformation parameter $\alpha$, on-site modulation strength $\lambda$, period $1 / b$, phase parameter $\phi$, and a constant offset value $\mu=2 t$. For a quasiperiodic modulation, we take an irrational $b=(\sqrt{5}-1) / 2$. Note that $\alpha=-1$ corresponds to a constant on-site energy, whereas $\alpha=0$ is a rescaled version of the AAH model. We only consider $|\alpha|<1$ so that the local potential is bounded. Diagonalization of the Hamiltonian in Eq. (1) gives $H=\sum_{k} \epsilon_{k} \eta_{k}^{\dagger} \eta_{k}$, where $\epsilon_{k}$ is the energy of the $k$ th single-particle eigenstate and $\eta_{k}\left(\eta_{k}^{\dagger}\right)$ being its corresponding eigenmode annihilation (creation) operator. Local modes are expressed via a unitary transformation $S$ as $a_{l}=\sum_{k} S_{l, k} \eta_{k}$. This model exhibits a single-particle mobility edge where eigenstates with an energy greater than $E_{\mathrm{mob}}=2 \lambda(|t / \lambda|-1) / \alpha+\mu$ are localized for any value of $\phi$ [19]. In the following we use units for which the tunneling $t$, the Boltzmann constant $k_{B}$, and the reduced Planck constant $\hbar$ are set to unity.

The chain is coupled at its edges, denoted as site $\ell=\{1, L\}$, to heat baths of noninteracting spins at different temperatures. For both baths the $\nu$ th spin has an energy $\varepsilon_{\nu}$ and couples to the system with a strength $g_{\nu}$ via a term $\sum_{\nu} g_{\nu}\left(a_{\ell}+a_{\ell}^{\dagger}\right)\left(\sigma_{\nu, \ell}^{+}+\sigma_{\nu, \ell}^{-}\right)$, where $\sigma_{\nu, \ell}^{+}$and $\sigma_{\nu, \ell}^{-}$are, respectively, the raising and lowering operators for the $\nu$ th spin coupled to site $\ell$. The evolution of the system's density matrix $\rho$ in time $\tau$ in the presence of the heat baths is modeled by a Lindblad master equation [34-38]

$$
\begin{aligned}
\frac{d \rho}{d \tau}= & -i[H, \rho] \\
& +\sum_{k, \ell}\left|S_{\ell, k}\right|^{2} J\left(\epsilon_{k}\right)\left[\left(1-n_{S}\left(\beta_{\ell} \epsilon_{k}\right)\right)\left(\eta_{k} \rho \eta_{k}^{\dagger}-1 / 2\left\{\eta_{k}^{\dagger} \eta_{k}, \rho\right\}\right)\right. \\
& \left.+n_{S}\left(\beta_{\ell} \epsilon_{k}\right)\left(\eta_{k}^{\dagger} \rho \eta_{k}-1 / 2\left\{\eta_{k} \eta_{k}^{\dagger}, \rho\right\}\right)\right],
\end{aligned}
$$

where $\beta_{\ell}=1 / T_{\ell}$ is the inverse temperature, $n_{S}\left(\beta_{\ell} \epsilon_{k}\right)=$ $\left(e^{\beta_{\ell} \epsilon_{k}}+1\right)^{-1}$ is the spin occupation factor of the bath coupled to the boundary site $\ell$, and $J(\epsilon)=\sum_{\nu} \pi\left|g_{\nu}\right|^{2} \delta\left(\epsilon-\varepsilon_{\nu}\right)$ is the spectral density of the baths. We consider an Ohmic spectral density $J(\epsilon) \propto \epsilon$.
For the NESS, the single particle density matrix is

$$
\left\langle\eta_{k}^{\dagger} \eta_{k}\right\rangle=\frac{\sum_{\ell}\left|S_{\ell, k}\right|^{2} n_{S}\left(\beta_{l} \epsilon_{k}\right)}{\sum_{\ell}\left|S_{\ell, k}\right|^{2}\left[1-\zeta n_{S}\left(\beta_{l} \epsilon_{k}\right)\right]},
$$

with $\zeta=2$ additionally reflecting the spin nature of the baths. This can be readily computed because the system is noninteracting and hence the one-body correlations form a close set of equations and can thus be solved exactly. Note that if only one bath is coupled to the system, since $n_{S}\left(\beta \epsilon_{k}\right) /\left(1-2 n_{S}\left(\beta \epsilon_{k}\right)\right)=n_{B}\left(\beta \epsilon_{k}\right)=\left(e^{\beta \epsilon_{k}}-1\right)^{-1}$, we recover the Bose occupation factor for each eigenstate $k$ with nonzero coupling to the bath, as expected. The steady state energy current $\mathcal{J}$ is then

$$
\begin{aligned}
\mathcal{J} & =\operatorname{Tr}\left[H \mathcal{D}_{1}(\rho)\right]=-\operatorname{Tr}\left[H \mathcal{D}_{L}(\rho)\right], \\
& =\sum_{k} \epsilon_{k}\left|S_{1, k}\right|^{2}\left|S_{L, k}\right|^{2} J\left(\epsilon_{k}\right) \frac{n_{S}\left(\beta_{1} \epsilon_{k}\right)-n_{S}\left(\beta_{L} \epsilon_{k}\right)}{\sum_{\ell}\left|S_{\ell, k}\right|^{2}\left[1-\zeta n_{S}\left(\beta_{\ell} \epsilon_{k}\right)\right]},
\end{aligned}
$$

where $\mathcal{D}_{l}(\cdot)$ is the superoperator acting on the density operator at site $l$, and is defined in Ref. [37]. In the following we numerically diagonalize the quadratic Hamiltonian (1) to compute the $S_{l, k}$. More details about the Hamiltonian and its spectrum can be found in Ref. [19].

We refer to forward bias $\left(\mathcal{J}_{f}\right)$ as the case in which the hotter bath, with temperature $T_{h}$, is coupled to the first site and the cold one, with temperature $T_{c}$, is coupled to the last, while reverse bias $\left(\mathcal{J}_{r}\right)$ is the opposite case. The magnitude of the rectification is signaled by the rectification coefficient, which is the ratio between the current in forward bias $\mathcal{J}_{f}$ and that in reverse bias $\mathcal{J}_{r}$,

$$
\mathcal{R}=-\frac{\mathcal{J}_{f}}{\mathcal{J}_{r}} .
$$

The rectification coefficient $\mathcal{R}=1$ when there is no rectification, while $\mathcal{R} \gg 1$ or $\mathcal{R} \ll 1$ signal strong rectification in one or the other direction. Importantly, if the baths had been bosonic the expression for the current in Eq. (4) would be identical except for replacing $n_{S}$ with $n_{B}$ and setting $\zeta=0$. It would then follow that the current is antisymmetric in the exchange of $\beta_{1}$ with $\beta_{L}$, and hence no rectification, as predicted in Refs. [13,14].

Results.-When $\lambda=0$ or $\alpha=-1$ the system corresponds to a uniform tight binding model; hence the forward and reverse currents are identical and there is no rectification. In Fig. 1(a) we report the rectification coefficient $\mathcal{R}$ with $\alpha$ for a sequence of increasing $\lambda$ 's. We observe that there are regions in the parameter space with very large rectifications, close to $\mathcal{R} \approx 100$. Moreover, as $\lambda$ increases, the range of $\alpha$ 's for which this strong rectification appears increases significantly. In the limit of $\lambda=1$, there is strong rectification for all $\alpha>0$, but beyond that value all states are localized and the system is an insulator in both 


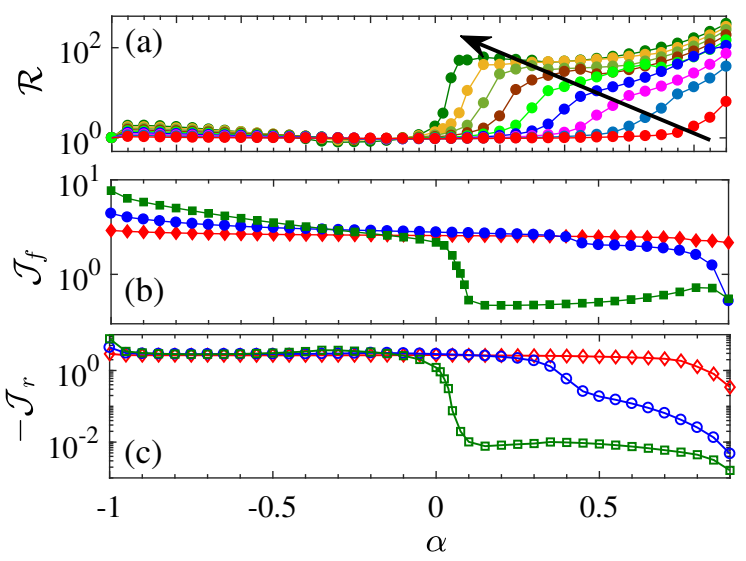

FIG. 1. (a) Rectification $\mathcal{R}$, (b) forward bias current $\mathcal{J}_{f}$, and (c) reverse bias current $\mathcal{J}_{r}$ versus the deformation parameter $\alpha$. In (a) different lines correspond to different values of $\lambda$, from 0.1 to 0.9 with steps of 0.1 , increasing in the direction of the arrow. (b)-(c) Forward and reverse bias currents for $\lambda=0.1$ (red $\diamond), 0.4$ (blue $\circ$ ), and 0.9 (green $\square$ ). $\mathcal{J}_{f}$ is represented by full symbols while $\mathcal{J}_{r}$ by empty symbols. Other parameters are chain length $L=1000$, phase $\phi=\pi$, and temperatures of the baths are $T_{h}=1000.1$ and $T_{c}=0.1$

directions. For negative $\alpha$ instead, and as long as $\lambda$ is positive, all states are delocalized and the rectification is small. In Figs. 1(b)-1(c) we show the currents in the forward and reverse bias, respectively. The regime of high rectification corresponds to a reduction in both the forward and reverse bias currents, but with substantially larger suppression in reverse bias.

In order to understand the role of the mobility edge, we study the rectification together with localization properties of the NESS. This is most easily revealed by the inverse participation ratio $\langle I\rangle=\sum_{k} I(k)\left\langle\eta_{k}^{\dagger} \eta_{k}\right\rangle$, where $\left\langle\eta_{k}^{\dagger} \eta_{k}\right\rangle$ is given by Eq. (3) and $I(k)=\sum_{l}\left|S_{l, k}\right|^{4} / \sum_{n}\left|S_{n, k}\right|^{2}$. The inverse participation ratio $I(k)$ for an eigenstate $k$ is closer to unity for more localized eigenstates, while it is of the order of $1 / L$ for delocalized ones. Hence, a NESS with highly occupied localized states will have a larger $\langle I\rangle$. In Fig. 2(a) we show $\langle I\rangle$ for the forward (filled symbols) and reverse (empty symbols) biases, and for different $\lambda$ 's. For these parameters, $\langle I\rangle$ for the reverse bias NESS is always larger than that of the forward bias. More importantly, at a particular value of $\alpha$, which changes with $\lambda$, both forward and reverse $\langle I\rangle$ grow significantly. As highlighted in Fig. 2(b) by dot-dashed lines, it is around these values of $\alpha$ that we also observe a significant increase in the rectification.

The relevance of the mobility edge in affecting the rectification can also be inferred in Fig. 2(c). Here we show a density plot of the fraction of localized single-particle eigenstates $f_{\text {loc }}$, where a state is considered localized if its energy is larger than the mobility edge $E_{\text {mob }}$ [19]. For several different $T_{h}$ we also plot the midpoint $\alpha$ where a significant uplift in $\mathcal{R}$ occurs as a function of $\lambda$, giving
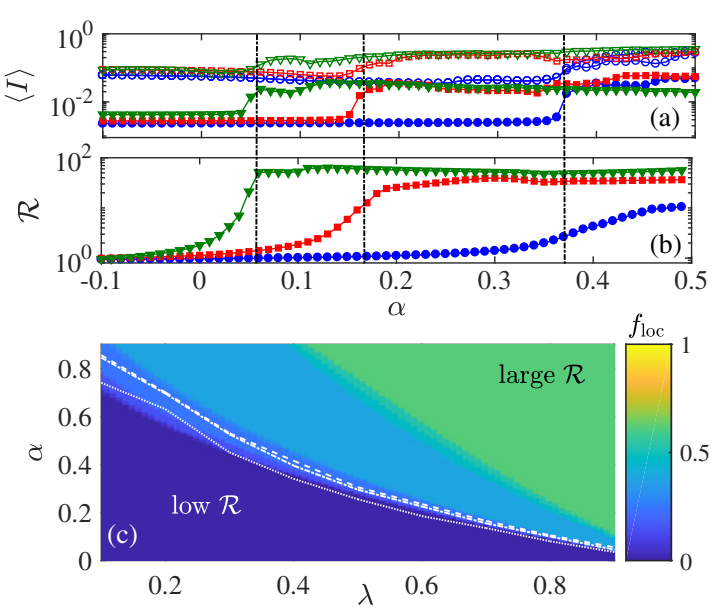

FIG. 2. (a) Inverse participation ratio $\langle I\rangle$ for forward (filled symbols) and reverse (empty symbols) bias versus deformation parameter $\alpha$ for $\lambda=0.4$ (blue empty circle), 0.7 (red empty square), and 0.9 (green empty triangle). (b) Rectification $\mathcal{R}$ versus $\alpha$ for $\lambda=0.4$ (blue circle), 0.7 (red square), and 0.9 (green triangle). In panels (a)-(b) we have used $T_{h}=1000+T_{c}$. (c) Density plot of fraction of localized states $f_{\text {loc }}$ as a function of $\alpha$ and $\lambda$. The white lines represent the values of $\alpha$ and $\lambda$ at which the rectification varies significantly. More precisely we consider $T_{h}=10.1$ (dotted line), $T_{h}=100.1$ (dot-dashed line), and $T_{h}=1000.1$ (dashed line). Common parameters are $L=1000, \phi=\pi$, and $T_{c}=0.1$. Black dot-dashed lines highlight the value of $\alpha$ at which the inverse participation ratio increases significantly.

curves that delineate the low and high rectification regimes. These curves clearly demonstrate that the $\alpha$ 's where rectification increases correspond closely with the values where there is a marked increase of localized states in the system. For $\lambda \approx 1$ we see large rectification for small $\alpha$ that is essentially independent of temperature, owing to the small energy threshold for localization. For smaller values of $\lambda$ a weak dependence on temperature is observed since $\mathcal{R}$ displays a broader slope, making the crossover less sharp. There is also a decreased sensitivity to the mobility edge for smaller $T_{h}$ as the highest energy eigenstates are less populated.

However the presence of a larger fraction of localized modes does not fully explain the phenomenology of this system. The link between rectification and localization is further unraveled by examining more closely the dependence of the rectification with the phase $\phi$ and the coupling of the baths to the system eigenstates. This parameter does not vary the energy spectrum nor the number of localized modes, but it shifts the position of these modes. Figure 3(a), which depicts $\mathcal{R}$ as a function of $\phi$, shows that by tuning $\phi$ it is possible to obtain rectification around $\mathcal{R} \approx 400$ for $\phi \approx 3$, and $\mathcal{R} \approx 1 / 400$ (i.e., strong rectification in the opposite direction) for $\phi \approx 5.5$. Hence the potential parameter $\phi$ can be used to control the direction of the rectification. In Fig. 3(b) we report $\max _{k}\left|S_{\ell, k}\right|$, i.e., the 


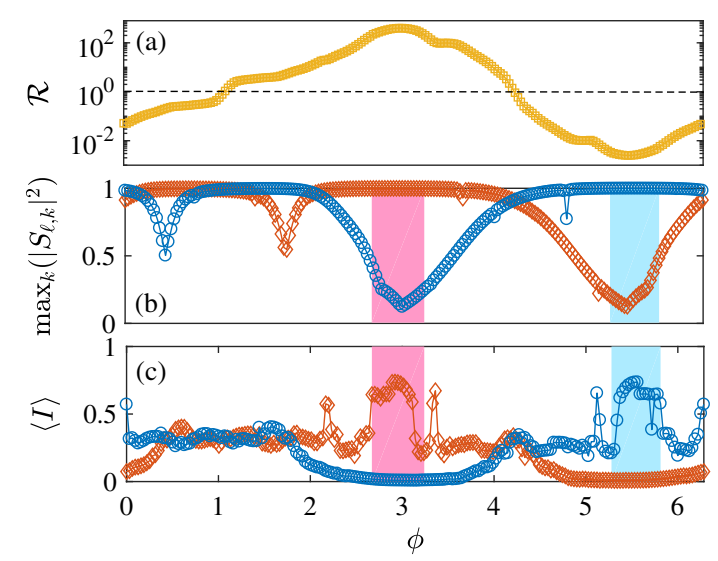

FIG. 3. (a) Rectification versus phase $\phi$ in the log-lin scale to highlight the regions in which the rectification is in different directions. (b) Maximum coupling magnitude of any $k$ mode to the first site (blue empty cirlce) or to the last site (red empty diamond). (c) Average inverse participation ratio $\langle I\rangle$ as a function of $\phi$ for the forward (blue empty circle) and reverse (red empty circle) bias. Parameters are $\alpha=\lambda=0.9, T_{h}=1000.1, T_{c}=0.1$.

maximum coupling strength of the bath at $\ell$ to any system eigenstate $k$. Owing to the unitarity of $S$ we have that $0 \leq \max _{k}\left|S_{\ell, k}\right| \leq 1$. Consequently, when $\max _{k}\left|S_{\ell, k}\right|$ approaches unity it indicates the coupling is dominated by one eigenstate, which for the spatially localized systembath interaction assumed here can only occur if that eigenstate is similarly localized. In contrast, a small value indicates couplings to numerous delocalized eigenstates. We see that $\mathcal{R} \approx 1$ whenever the maximum coupling is similar at both edges. However, our key finding is that strong rectification $\mathcal{R} \gg 1$ (or $\mathcal{R} \ll 1$ ) manifests when one bath couples mainly to a single highly localized eigenstate at the boundary, while the other bath is coupled to many delocalized eigenstates. This is demonstrated by the two disjoint shaded regions (pink for the bath at $\ell=L$ and light blue for $\ell=1$ ) in Figs. 3(b)-3(c), which signify the phases $\phi$ where $I(k) \approx 1$ for the maximally coupled eigenstate. In Fig. 3(c) we show the average inverse participation ratio $\langle I\rangle$ of the NESS in forward (blue $\circ$ ) and reverse bias (red $\diamond$ ). Again we observe that in the regions of strongest rectification the NESS is highly delocalized in one bias and strongly localized in the other [39].

Strong rectification is therefore a consequence of this disparity in bath eigenstate couplings $S_{\ell, k}$, combined with the difference in statistics of the baths and system that is reflected in the form of the denominator of Eq. (4). To understand this intuitively, suppose $T_{c}=0$ temperature while $T_{h}=\infty$ so that $n_{S}\left(\beta_{\ell} \epsilon_{k}\right) \approx 1 / 2$ for the modes coupled to the hot bath and $n_{S}\left(\beta_{\ell} \epsilon_{k}\right)=0$ for the cold bath. In this scenario Eq. (4) gives a significantly larger current when the cold bath is coupled to a localized mode compared to when the hot bath is coupled to a localized mode [40]. We stress that since the system has an extensive

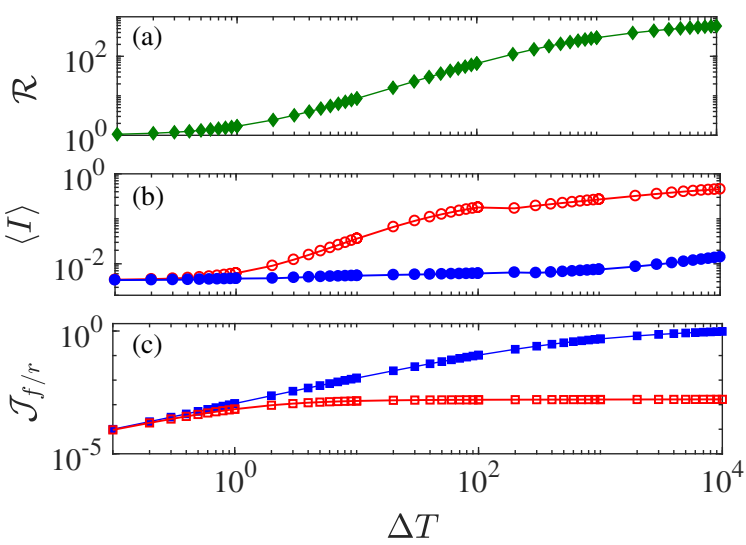

FIG. 4. (a) Rectification $\mathcal{R}$ versus temperature difference $\Delta T$. (b) Inverse participation ratio $\langle I\rangle$ and (c) steady state currents for forward $\mathcal{J}_{f}$ (filled blue symbols) and reverse $\mathcal{J}_{f}$ (empty red symbols) biases, versus $\Delta T$. Common parameters are $\alpha=\lambda=0.9, L=1000, \phi=\pi$, and $T_{c}=1$.

large number of localized modes, this result is not due to fine tuning of the potential parameters.

The rectification effect outlined here is observed over a wide range of temperatures. When temperature increases there are two main contributions: the bias which drives the current increases, so the current can increase, and the population of localized higher energy eigenmodes also increases. Given the different occupation of localized and delocalized modes between the forward and the reverse bias, rectification increases with larger temperature difference $\Delta T$. We analyze this in Fig. 4. Specifically, we show the rectification $\mathcal{R}$, average inverse participation ratio $\langle I\rangle$, and the forward and reverse currents $\mathcal{J}_{f / r}$, as a function of $\Delta T$ in Figs. 4(a)-4(c), respectively. For the reverse bias [empty symbols in panels (b)-(c)], $\langle I\rangle$ saturates to a larger value at lower $\Delta T$ compared to the forward bias [full symbols in panels (b)-(c)]. Consequently, a large gap between $\mathcal{J}_{f}$ and $\mathcal{J}_{r}$ opens up as $\Delta T$ increases and the rectification grows to $\mathcal{R} \approx 580$. While large rectification occurs for such extreme temperature differences, Fig. 4(a) nonetheless shows sizable rectifications for much lower temperatures. Further analysis on how the phase $\phi$ and the bath temperatures affect the localization and transport properties of the steady state can be found in the Supplemental Material [37].

Conclusions. - We have studied how a quadratic bosonic system with a mobility edge coupled to spin baths can rectify energy current. While the difference in particle statistics is fundamental in order to achieve rectification, we found regimes of strong rectification and identified their emergence as a result of one bath being strongly coupled to a highly localized mode while the other bath couples broadly to many delocalized modes. Strong rectification thus emerges due to the presence of a mobility edge, and it can be tuned by shifting the mobility edge or by tuning the location of the strongly localized modes. 
Crucially, since the system is ballistic, the current accompanying this large rectification coefficient remains appreciable even as the system size increases, in contrast, for example, to diffusive systems where the temperature gradients decrease with $L$. The effect observed should occur for other systems with different statistics in the bath and the system, and mobility edges, such as a quasiperiodically modulated $X X$ spin chain coupled to bosonic baths. Future work includes studying the effect of strong coupling to the baths, as well as considering the role of many-body interactions. For the setup considered, the interactions between the bosons could be induced by an atom trapped in each cavity, while for the current setup the photons would be noninteracting.

D. P. and V. B. are grateful to G. Benenti for insightful discussions. D. P. and V. B. acknowledge support from the Singapore Ministry of Education AcRF MOE Tier-II (project MOE2016-T2-1-065, WBS R-144-000-350-112). S. R. C. gratefully acknowledges support from the UK's Engineering and Physical Sciences Research Council (EPSRC) under Grant No. EP/P025110/1. J. G. is supported by an SFI-Royal Society University Research Fellowship. J.G. acknowledges that this project has received funding from the European Research Council (ERC) under the European Unions Horizon 2020 research and innovation programme (Grant Agreement No. 758403). The computational work for this Letter was partially performed on resources of the National Supercomputing Centre, Singapore [41].

[1] G. Benenti, G. Casati, K. Saito, and R. S. Whitney, Phys. Rep. 694, 1 (2017).

[2] F. Binder, L. A. Correa, C. Gogolin, J. Anders, and G. Adesso, Thermodynamics in the Quantum Regime (Springer, New York, 2018).

[3] M. Terraneo, M. Peyrard, and G. Casati, Phys. Rev. Lett. 88, 094302 (2002).

[4] B. Li, L. Wang, and G. Casati, Phys. Rev. Lett. 93, 184301 (2004).

[5] N. Li, J. Ren, L. Wang, G. Zhang, P. Hänggi, and B. Li, Rev. Mod. Phys. 84, 1045 (2012).

[6] T. Werlang, M. A. Marchiori, M. F. Cornelio, and D. Valente, Phys. Rev. E 89, 062109 (2014).

[7] L. Zhang, Y. Yan, C.-Q. Wu, J.-S. Wang, and B. Li, Phys. Rev. B 80, 172301 (2009).

[8] G. T. Landi, E. Novais, M. J. de Oliveira, and D. Karevski, Phys. Rev. E 90, 042142 (2014).

[9] L. Schuab, E. Pereira, and G. T. Landi, Phys. Rev. E 94, 042122 (2016).

[10] V. Balachandran, G. Benenti, E. Pereira, G. Casati, and D. Poletti, Phys. Rev. E 99, 032136 (2019).

[11] V. Balachandran, G. Benenti, E. Pereira, G. Casati, and D. Poletti, Phys. Rev. Lett. 120, 200603 (2018).

[12] L. Arrachea, G. S. Lozano, and A. A. Aligia, Phys. Rev. B 80, 014425 (2009).
[13] L.-A. Wu and D. Segal, Phys. Rev. Lett. 102, 095503 (2009)

[14] L.-A. Wu, C. X. Yu, and D. Segal, Phys. Rev. E 80, 041103 (2009).

[15] P. W. Anderson, Phys. Rev. 109, 1492 (1958).

[16] S. Aubry and G. André, Ann. Isr. Phys. Soc. 3, 133 (1980).

[17] P. G. Harper, Proc. Phys. Soc. London Sect. A 68, 874 (1955).

[18] J. Biddle and S. D. Sarma, Phys. Rev. Lett. 104, 070601 (2010).

[19] S. Ganeshan, J. H. Pixley, and S. D. Sarma, Phys. Rev. Lett. 114, 146601 (2015).

[20] G. Roati, C. D’Errico, L. Fallani, M. Fattori, C. Fort, M. Zaccanti, G. Modugno, M. Modugno, and M. Inguscio, Nature (London) 453, 895 (2008).

[21] C. D’Errico, E. Lucioni, L. Tanzi, L. Gori, G. Roux, I. P. McCulloch, T. Giamarchi, M. Inguscio, and G. Modugno, Phys. Rev. Lett. 113, 095301 (2014).

[22] M. Schreiber, S. S. Hodgman, P. Bordia, H. P. Lüschen, M. H. Fischer, R. Vosk, E. Altman, U. Schneider, and I. Bloch, Science 349, 842 (2015).

[23] P. Bordia, H. P. Lüschen, S. S. Hodgman, M. Schreiber, I. Bloch, and U. Schneider, Phys. Rev. Lett. 116, 140401 (2016).

[24] P. Bordia, H. Lüschen, U. Schneider, M. Knap, and I. Bloch, Nat. Phys. 13, 460 (2017).

[25] P. Bordia, H. Lüschen, S. Scherg, S. Gopalakrishnan, M. Knap, U. Schneider, and I. Bloch, Phys. Rev. X 7, 041047 (2017).

[26] H. P. Lüschen, S. Scherg, T. Kohlert, M. Schreiber, P. Bordia, X. Li, S. D. Sarma, and I. Bloch, Phys. Rev. Lett. 120, 160404 (2018).

[27] F. A. An, E. J. Meier, and B. Gadway, Phys. Rev. X 8, 031045 (2018)

[28] T. Kohlert, S. Scherg, X. Li, H. P. Lüschen, S. D. Sarma, I. Bloch, and M. Aidelsburger, Phys. Rev. Lett. 122, 170403 (2019).

[29] A. Purkayastha, A. Dhar, and M. Kulkarni, Phys. Rev. B 96, 180204(R) (2017).

[30] U. Sivan and Y. Imry, Phys. Rev. B 33, 551 (1986).

[31] F. Badshah, S. Qamar, and M. Paternostro, Phys. Rev. A 90, 033813 (2014).

[32] M. A. Norcia, R. J. Lewis-Swan, J. R. K. Cline, B. Zhu, A. M. Rey, and J. K. Thompson, Science 361, 259 (2018).

[33] The quasiperiodic potential can be expanded as the cosine Fourier series $V_{l}=a_{0}+\sum_{n=1}^{\infty} a_{n} \cos [n(2 \pi l b+\phi)]$, where $a_{0}=\mu+g-2 \lambda \cosh \beta, \quad a_{n}=2 g e^{-\beta n}$ with $g=$ $[2 \lambda(1+\cosh \beta) / \tanh \beta], \cosh \beta=1 / \alpha$ and using the identity $\sinh \beta /[\cosh \beta-\cos (2 \pi l b+\phi)]=\sum_{n=-\infty}^{\infty} e^{-\beta|n|} e^{i n(2 \pi l b+\phi)}$.

[34] G. Lindblad, Commun. Math. Phys. 48, 119 (1976).

[35] V. Gorini, A. Kossakowski, and E. C. G. Sudarshan, J. Math. Phys. (N.Y.) 17, 821 (1976).

[36] H.-P. Breuer and F. Petruccione, The Theory of Open Quantum Systems (Oxford University Press, Oxford, 2007).

[37] See Supplemental Material at http://link.aps.org/ supplemental/10.1103/PhysRevLett.123.020603 for more detailed analysis of the role of system and bath parameters on the rectification mechanism and the derivation of the expression of current in Eq. (4). 
[38] The use of a Lindblad master equation requires a small coupling to the baths and fast decay of the bath correlations. We shall note, though, that within this approach the results are independent, up to a global factor, of the strength of the coupling to the baths.

[39] The apparent noisy features of Fig. 3 are due to the quasiperiodic nature of the potential used.

[40] For a nonzero current both baths must couple to at least one common eigenstate $K$, which is necessarily delocalized in order to have an overlap with both boundaries. For the scenario described the bath at $\ell=L$ has a coupling dominated by a localized eigenstate while the one at $\ell=1$ is coupled to delocalized eigenstates. This is modeled by taking $S_{L, K}=q$ and $S_{1, K}=Q$ with $Q \gg q$. For $T_{h}=\infty$ and $T_{c}=0$ Eq. (4) gives $\mathcal{J}_{f}=\frac{1}{2} \epsilon_{K} J\left(\epsilon_{K}\right) Q^{2}$ in forward bias and $\mathcal{J}_{r}=-\frac{1}{2} \epsilon_{K} J\left(\epsilon_{K}\right) q^{2}$ in reverse bias. Thus $\mathcal{R}=$ $(Q / q)^{2} \gg 1$.

[41] https://www.nscc.sg/.

Correction: The previously published Figure 4 was set with incorrect part labels during the production cycle and has been fixed. 